\title{
COEXISTENCE OF LIMIT CYCLES AND HOMOCLINIC LOOPS IN A SIRS MODEL WITH A NONLINEAR INCIDENCE RATE*
}

\author{
YILEI TANG ${ }^{\dagger}$, DEQING HUANG‡, SHIGUI RUAN§, AND WEINIAN ZHANG‡
}

\begin{abstract}
Recently, Ruan and Wang [J. Differential Equations, 188 (2003), pp. 135-163] studied the global dynamics of a SIRS epidemic model with vital dynamics and a nonlinear saturated incidence rate. Under certain conditions they showed that the model undergoes a Bogdanov-Takens bifurcation; i.e., it exhibits saddle-node, Hopf, and homoclinic bifurcations. They also considered the existence of none, one, or two limit cycles. In this paper, we investigate the coexistence of a limit cycle and a homoclinic loop in this model. One of the difficulties is to determine the multiplicity of the weak focus. We first prove that the maximal multiplicity of the weak focus is 2 . Then feasible conditions are given for the uniqueness of limit cycles. The coexistence of a limit cycle and a homoclinic loop is obtained by reducing the model to a universal unfolding for a cusp of codimension 3 and studying degenerate Hopf bifurcations and degenerate Bogdanov-Takens bifurcations of limit cycles and homoclinic loops of order 2.
\end{abstract}

Key words. degenerate Bogdanov-Takens bifurcation, degenerate Hopf bifurcation, limit cycle, homoclinic loop, revised sign list

AMS subject classifications. 34C23, 92D30

DOI. $10.1137 / 070700966$

1. Introduction. Periodic oscillations are common phenomena observed in the incidence of many infectious diseases such as chickenpox, influenza, measles, mumps, rubella, etc. (see Hethcote [10, 11], Hethcote and Levin [12], Hethcote, Stech, and van den Driessche [13]). It is very important to understand such epidemic patterns in order to introduce public health interventions and control the spread of diseases. Recent studies have demonstrated that the incidence rate plays a crucial role in producing periodic oscillations in epidemic models (Alexander and Moghadas [1, 2], Derrick and van den Driessche [6], Hethcote and van den Driessche [14], Liu et al. [17, 18], Lizana and Rivero [19], Moghadas [21], Moghadas and Alexander [22], Ruan and Wang [25], Wang $[26])$.

In most epidemic models (see Anderson and May [3]), the incidence rate (the number of new cases per unit time) takes the mass-action form with bilinear interactions, namely, $\kappa S(t) I(t)$, where $S(t)$ and $I(t)$ are the numbers of susceptible and infectious individuals at time $t$, respectively, and the constant $\kappa$ is the probability of transmission per contact. Epidemic models with such bilinear incidence rates usually have at most one endemic equilibrium and do not exhibit periodicity; the disease will be eradicated if the basic reproduction number is less than one and will persist otherwise (Anderson and May [3], Hethcote [11]). There are many reasons for using nonlinear incidence rates, and various forms of nonlinear incidence rates have

* Received by the editors August 23, 2007; accepted for publication (in revised form) August 5, 2008; published electronically December 3, 2008.

http://www.siam.org/journals/siap/69-2/70096.html

$\dagger$ Department of Mathematics, Shanghai Jiao Tong University, Shanghai 200240, China and Department of Mathematics, Sichuan University, Chengdu, Sichuan 610064, China (mathtyl@163.com).

${ }^{\ddagger}$ Department of Mathematics, Sichuan University, Chengdu, Sichuan 610064, China (toglyhdq@ sina.com, matzwn@126.com). The work of the fourth author was supported by NSFC (China) grants 10571127 and 10825104 and SRFDP 20050610003.

$\S$ Department of Mathematics, University of Miami, Coral Gables, FL 33124-4250 (ruan@math. miami.edu). This author's research was supported by NSF grant DMS-0715772.

621 
been proposed recently. For example, in order to incorporate the effect of behavioral changes, Liu, Levin, and Iwasa [18] used a nonlinear incidence rate of the form

$$
g(I) S=\frac{\kappa I^{\ell} S}{1+\alpha I^{h}},
$$

where $\kappa I^{\ell}$ measures the infection force of the disease, $1 /\left(1+\alpha I^{h}\right)$ describes the inhibition effect from the behavioral change of the susceptible individuals when the number of infectious individuals increases, $\ell, h$, and $\kappa$ are all positive constants, and $\alpha$ is a nonnegative constant. See also Alexander and Moghadas [1, 2], Derrick and van den Driessche [6], Hethcote and van den Driessche [14], Moghadas [21], etc. Notice that the bilinear interaction is a special case of (1.1) with $\alpha=0$ and $\ell=1$.

The nonlinear function $g(I)$ given by (1.1) includes three types. (a) Unbounded incidence function: $\ell>h$. The case when $\ell=h+1$ was considered by Hethcote and van den Driessche [14]. The function is unbounded as the bilinear incidence rate (see Figure 1(a)). (b) Saturated incidence function: $\ell=h$. The case when $\ell=h=1$, i.e., $g(I)=\kappa I /(1+\alpha I)$, was proposed by Capasso and Serio [5] to describe a "crowding effect" or "protection measures" in modeling the cholera epidemics in Bari in 1973. A similar type of sigmoidal function was also used to represent doseresponse relationships observed in parasite infection experiments (Regoes, Ebert, and Bonhoeffer [23]). The function tends to a saturation level as the number of infectious individuals $I$ becomes large (see Figure 1(b)). (c) Nonmonotone incidence function: $\ell<h$. Such functions can be used to interpret the "psychological effects" (Capasso and Serio [5]): for a very large number of infectious individuals the infection force may decrease as the number of infectious individuals increases (see Figure 1(c)), because in the presence of a large number of infectious individuals the population may tend to reduce the number of contacts per unit time, as seen with the spread of SARS (see Wang [26], Xiao and Ruan [28]).

From the graphs in Figure 1, one would expect that the dynamics of epidemic models with unbounded incidence rates are similar to those with bilinear incidence rates. In fact, Hethcote and van den Driessche [14] found that in a SEIRS model with $\ell=h+1$, the classical threshold results hold; namely, the disease dies out below the threshold, and the disease level approaches the endemic equilibrium above the threshold. For a SIRS model with the nonmonotone incidence function $g(I)=$ $k I /\left(1+\alpha I^{2}\right)$, Xiao and Ruan [28] demonstrated that either the number of infectious individuals tends to zero as time evolves or the disease persists. We conjecture that the dynamics of SIRS models with nonmonotone incidence rates are similar to those observed by Xiao and Ruan [28].

On the other hand, the dynamics of epidemic models with saturated incidence rates (when $\ell=h$ ) have been shown to be very rich and complex. For a SEIRS model with $\ell=h$, Hethcote and van den Driessche [14] observed that the threshold concept becomes more complicated since the asymptotic behavior can depend on both the threshold and the initial values. The model can have none, one, or two endemic equilibria, and the disease can die out above the threshold for some initial values. Periodic solutions appear through Hopf bifurcation. The results are analogous to those obtained by Liu, Hethcote, and Levin [17] for SIRS models with $\ell=h$. The case $\ell=h=1$ has been discussed briefly by Capasso and Serio [5] and recently in some detail by Gomes et al. [8], who obtained the existence of backward bifurcations, oscillations, and Bogdanov-Takens points in SIR and SIS models. These indicate that the case when $\ell=h \geq 2$ can be very complicated and deserves further investigation. 


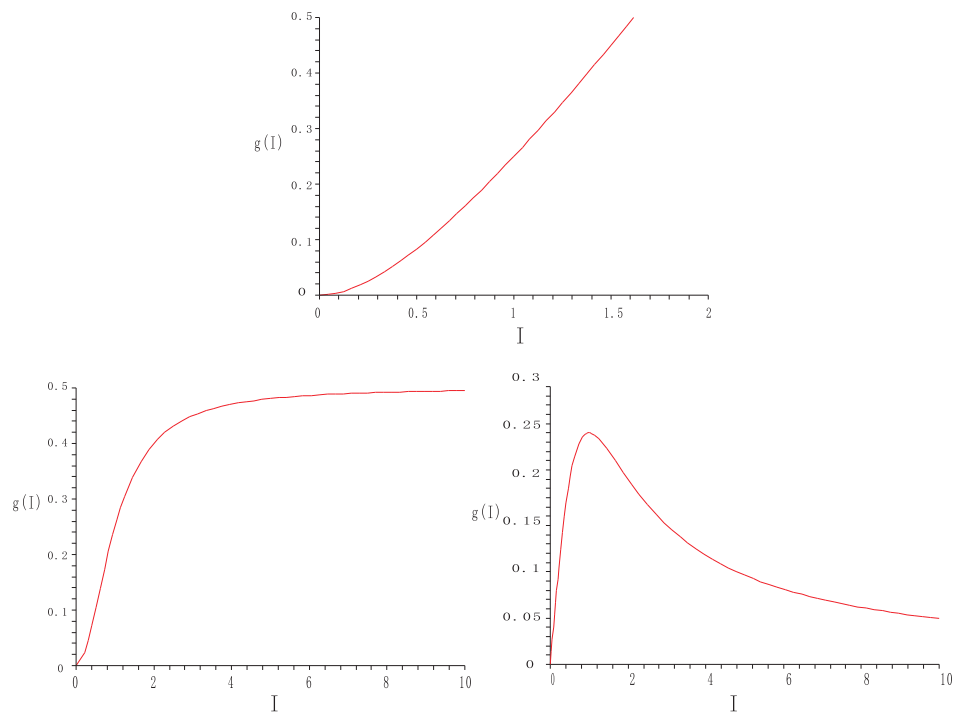

FIG. 1. Graphs of the incidence function $g(I)=\frac{k I^{\ell}}{1+\alpha I^{h}}$. (a) Top: Unbounded incidence when $\ell>h(\ell=2, h=1)$. (b) Bottom-left: Saturated incidence when $\ell=h(\ell=h=2)$. (c) Bottom-right: Nonmonotone incidence when $\ell<h(\ell=1, h=2)$. Here, $k=0.5, \alpha=1$.

In order to better understand the generic bifurcations in SIRS models with saturated incidence rates and also motivated by the work of Liu et al. [17, 18] and Hethcote and van den Driessche [14], Ruan and Wang [25] studied the global dynamics of a SIRS model with the nonlinear incidence function $g(I)=\kappa I^{2} /\left(1+\alpha I^{2}\right)$, i.e., $\ell=h=2$ :

$$
\left\{\begin{aligned}
\frac{d S}{d t} & =b-\delta S-\frac{\kappa I^{2} S}{1+\alpha I^{2}}+\nu R \\
\frac{d I}{d t} & =\frac{\kappa I^{2} S}{1+\alpha I^{2}}-(\delta+\gamma) I \\
\frac{d R}{d t} & =\gamma I-(\delta+\nu) R
\end{aligned}\right.
$$

where $b>0$ is the recruitment rate of the population, $\delta>0$ is the death rate of the population, $\gamma>0$ is the recovery rate of infectious individuals, and $\nu>0$ is the rate of removed individuals who lose immunity and return to the susceptible class. Summing up the three equations in (1.2), we obtain an equation $d N / d t=b-\delta N$ with $N(t)=S(t)+I(t)+R(t)$. Obviously, all solutions of this equation tend to its equilibrium $N(t) \equiv N_{0}=b / \delta$ as $t \rightarrow+\infty$. Thus, all important dynamical behaviors of system (1.2) occur on the plane $S+I+R=N_{0}$, and the restricted system on the plane becomes

$$
\left\{\begin{array}{l}
\frac{d I}{d t}=\frac{\kappa I^{2}}{1+\alpha I^{2}}\left(N_{0}-I-R\right)-(\delta+\gamma) I, \\
\frac{d R}{d t}=\gamma I-(\delta+\nu) R .
\end{array}\right.
$$

Under certain conditions Ruan and Wang [25] showed that the simplified model (1.3) undergoes a Bogdanov-Takens bifurcation; i.e., it exhibits saddle-node, Hopf, and homoclinic bifurcations. They also established the existence of none, one, or two limit cycles by applying the Bendixson-Dulac criterion [32], the Poincaré-Bendixson 
theorem [9], and a classic method for uniqueness of limit cycles in the Liénard equation [32], respectively. The coexistence (Theorem 2.9 in [25]) of two limit cycles is obtained by assuming that the successor function [32] (denoted by $d$ in [25]) can switch its signs.

In Ruan and Wang [25] the uniqueness of limit cycles was obtained under the assumption that a polynomial $h(x)$ of degree 6 is nonpositive for all $x$ in a definite interval, which is actually not easy to check. Moreover, only the first order Liapunov value of the weak focus $\left(I_{2}, R_{2}\right)$ in Theorem 2.6 of [25] was calculated. To have a better understanding of the dynamics of the system, we need to calculate higher order Liapunov values of the weak focus, which is difficult in general. In fact, the weak focus $\left(I_{2}, R_{2}\right)$ in Theorem 2.6 of [25] ( $E_{+}$in this paper) may have multiplicity 2, two limit cycles may arise from a degenerate Hopf bifurcation, and a limit cycle and a homoclinic loop may coexist via the degenerate Bogdanov-Takens bifurcation.

The study of model (1.3) is interesting and significant since it exhibits different and complicated dynamics such as periodic solutions, homoclinic orbits, multiple endemic equilibria, etc. The global dynamics is still not well understood. In this paper we further study the dynamical behavior of system (1.3). By rescaling the variables

$$
x=\left(\sqrt{\frac{\kappa}{\delta+\nu}}\right) I, \quad y=\left(\sqrt{\frac{\kappa}{\delta+\nu}}\right) R, \quad d \tau=(\delta+\nu) d t /\left(1+p I^{2}\right)
$$

and parameters

$$
p=\frac{\alpha(\delta+\nu)}{\kappa}, \quad A=N_{0} \sqrt{\frac{\kappa}{\delta+\nu}}, \quad m=\frac{\delta+\gamma}{\delta+\nu}, \quad q=\frac{\gamma}{\delta+\nu},
$$

system (1.3) is transformed into an equivalent system

$$
\begin{cases}\frac{d I}{d t}=-I\left[(m p+1) I^{2}+(R-A) I+m\right] & =: \mathcal{I}(I, R), \\ \frac{d R}{d t}=\left(1+p I^{2}\right)(q I-R) & =: \mathcal{R}(I, R),\end{cases}
$$

where we still use $I, R, t$ to present $x, y, \tau$ for simplicity and $I, R \geq 0, A, m, p, q>0$. We first calculate the second order Liapunov value at the weak focus and prove that the maximal multiplicity of the weak focus is 2 by technically dealing with some complicated multivariable polynomials, which implies that at most two limit cycles can arise near the weak focus. Then, by reducing the determination of the sign for polynomials of higher degrees to revised sign lists [31], we give some clean conditions on the parameters for the uniqueness of limit cycles. Finally, we reduce system (1.4) to a form of universal unfolding for a cusp of codimension 3 so as to give the bifurcation surfaces and display all limit cycles and homoclinic loops of order up to 2, from which the coexistence of limit cycles and homoclinic loops is established.

The paper is organized as follows. Some preliminary results on the existence and properties of equilibria are reviewed in section 2. Section 3 is devoted to the study of degenerate Hopf bifurcation. The uniqueness of limit cycles is considered in section 4 . In section 5, we study the degenerate Bogdanov-Takens bifurcation of the model. A brief discussion on the models, motivations, methods, and results is given in section 6 .

2. Preliminaries. We first recall some known results on the existence of equilibria. As shown in Ruan and Wang [25], system (1.4) has at most three equilibria $O=(0,0), E_{-}=\left(I_{-}, R_{-}\right)$, and $E_{+}=\left(I_{+}, R_{+}\right)$in the first quadrant, where

$$
I_{ \pm}=\frac{A \pm\left(A^{2}-4 m(m p+q+1)\right)^{1 / 2}}{2(m p+q+1)}, \quad R_{ \pm}=q I_{ \pm}
$$


It is easy to see that $O$ is the disease-free equilibrium of system (1.4) and is a stable node. Moreover, there are no positive equilibria if $A^{2}<4 m(m p+q+1)$ and two positive ones $E_{-}$and $E_{+}$if $A^{2}>4 m(m p+q+1)$. They coincide at $E_{0}=\left(I_{0}, R_{0}\right)=$ $(A / 2(m p+q+1), q A / 2(m p+q+1))$ if $A^{2}=4 m(m p+q+1)$. It is indicated in [25] that $E_{-}$is a saddle and $E_{+}$is a node, a focus, or a center. Moreover, the following results are given in Theorem 2.1 in [25].

LEMma 2.1. The equilibrium $E_{+}$is stable if one of the following inequalities holds:

$$
A^{2}>A_{c}^{2}, \quad m \leq 1, q<\frac{2 m p+1}{m-1}
$$

where

$$
A_{c}^{2}=: \frac{\left(m q+2 m-1-q+2 m^{2} p\right)^{2}}{(m-1)(m p+p+1)}
$$

$E_{+}$is unstable if

$$
A^{2}<A_{c}^{2}, m>1, \text { and } q>\frac{2 m p+1}{m-1} .
$$

When the parameters lie in the region

$$
\Omega=\left\{(A, m, p, q) \mid m>1, q>(2 m p+1) /(m-1), A^{2}=A_{c}^{2}\right\},
$$

the linearization of system (1.4) at $E_{+}$has a pair of purely imaginary eigenvalues. Let

$$
\mu=(1+2 m-q(m-1))+\left(4+2 m+4 q-6 m q+6 m^{2}+2 m^{2} q\right) p+4 m\left(m^{2}+2\right) p^{2}
$$

The following results on Hopf bifurcation are given in Theorem 2.6 in [25].

Lemma 2.2. Suppose that conditions in $\Omega$ hold. If $\mu<0$, then there is a stable periodic orbit in (1.4) as $A^{2}$ decreases from $A_{c}^{2}$. If $\mu>0$, there is an unstable periodic orbit in (1.4) as $A^{2}$ increases from $A_{c}^{2}$. If $\mu=0$, a Hopf bifurcation with codimension 2 may occur.

Obviously, in [25] a question remains open: Is $E_{+}$possibly a center when $\mu=0$ ? A negative answer will be given in section 3 . Regarding $\mu$ as a quadratic polynomial of $p$, we can easily see that the case $\mu=0$ happens if and only if the discriminant of $(2.2)$ is $\geq 0$.

As shown previously, when $A=A_{0}=: 2 \sqrt{m(m p+q+1)}$, the equilibrium $E_{0}$ appears in the interior of the first quadrant and is degenerate because the Jacobian matrix of the linearized system of (1.4) at $E_{0}$ has determinant 0.

Lemma 2.3. When $A=A_{0}, E_{0}$ is either a saddle-node if $p \neq((m-1) q-1) /(2 m)$ or a cusp otherwise.

Proof. For $p=((m-1) q-1) /(2 m)$ it was proved in [25] that system (1.4) has a cusp at $E_{0}$. Consider the case that $p \neq((m-1) q-1) /(2 m)$. With the change of variables $(I, R) \mapsto(x, y)$ defined by

$$
x=-\frac{(1+q+2 m p)\left(I-I_{0}\right)}{1+q+m p}+\frac{m\left(R-R_{0}\right)}{1+q+m p}, \quad y=-\frac{m q\left(I-I_{0}\right)}{1+q+m p}+\frac{m\left(R-R_{0}\right)}{1+q+m p},
$$

Copyright (c) by SIAM. Unauthorized reproduction of this article is prohibited. 
system (1.4) is rewritten as

$$
\left\{\begin{aligned}
\dot{x}= & \frac{-d_{0} \sqrt{1+m p+q} \sqrt{m}}{\left(b_{0} q-d_{0}\right)^{2}} x^{2}+\frac{2\left(p q b_{0}^{2}+b_{0} d_{0}+b_{0} d_{0} m p-b_{0} p d_{0}+d_{0}^{2}\right) \sqrt{m}}{b_{0} \sqrt{1+m p+q}\left(b_{0} q-d_{0}\right)^{2}} x y \\
& -\frac{\left(2 p q b_{0}^{2}-b_{0} d_{0} q+b_{0} d_{0}-2 b_{0} p d_{0}+b_{0} d_{0} m p+2 d_{0}^{2}\right) \sqrt{m}}{b_{0} \sqrt{1+m p+q}\left(b_{0} q-d_{0}\right)^{2}} y^{2}+O\left(|(x, y)|^{3}\right)=: X_{1}(x, y), \\
\dot{y}= & \left(d_{0}-b_{0} q\right) y-\frac{b_{0} q \sqrt{1+m p+q} \sqrt{m}}{\left(b_{0} q-d_{0}\right)^{2}} x^{2}+\frac{2\left(b_{0} q+q m p b_{0}+q p b_{0}+q d_{0}-p d_{0}\right) \sqrt{m}}{\sqrt{1+m p+q}\left(b_{0} q-d_{0}\right)^{2}} x y \\
& -\frac{\left(-b_{0} q^{2}+b_{0} q+q m p b_{0}+2 q p b_{0}+2 q d_{0}-2 p d_{0}\right) \sqrt{m}}{\sqrt{1+m p+q}\left(b_{0} q-d_{0}\right)^{2}} y^{2}+O\left(|(x, y)|^{3}\right)=: Y_{1}(x, y)
\end{aligned}\right.
$$

where $b_{0}=-m /(1+q+m p), d_{0}=-(1+q+2 m p) /(1+q+m p)$, and $E_{0}$ is translated to the origin. By the implicit function theorem, there is a unique function $y=\varsigma(x)$ such that $\varsigma(0)=0$ and $Y_{1}(x, \varsigma(x))=0$. Actually, we can solve from $Y_{1}(x, y)=0$ that

$$
\varsigma(x)=-\frac{q b_{0} \sqrt{1+q+m p} \sqrt{m}}{\left(b_{0} q-d_{0}\right)^{3}} x^{2}+O\left(|x|^{3}\right) .
$$

Substituting $y=\varsigma(x)$ into the first equation of (2.3), we get

$$
\dot{x}=X_{1}(x, \varsigma(x))=-\frac{d_{0} \sqrt{1+q+m p} \sqrt{m}}{\left(b_{0} q-d_{0}\right)^{2}} x^{2}+O\left(|x|^{3}\right) .
$$

Theorem 7.1 in Chapter 2 of [32] implies that the origin is a saddle-node of system (2.3). Thus, $E_{0}$ is a saddle-node of system (1.4).

3. Degenerate Hopf bifurcation. This section is a complement to the Hopf bifurcation analysis in Ruan and Wang [25]. In Lemma 2.2 the sign of $\mu$ is the same as the sign of the first Liapunov value of (1.4) if $E_{+}$is a weak focus, but [25] does not determine the sign of the higher order Liapunov values and whether $E_{+}$is a center. In this section, we overcome some technical difficulties in the computation of the higher order Liapunov values and prove that $E_{+}$is a weak focus of multiplicity at most 2 .

As in section 2, we consider those parameters in the region $\Omega$, defined in (2.1), where the Jacobian matrix at $E_{+}$has a pair of purely imaginary eigenvalues and the parameters $A, m, p$, and $q$ satisfy $A^{2}=A_{c}^{2}>4 m(m p+q+1)$. In this case, the first coordinate of $E_{+}$takes the form $I_{+}=\left(2 m^{2} p-q+m q-1+2 m\right) /(A(m p+p+$ 1)). A simple transformation $(I, R) \mapsto(x, y)$, which translates $E_{+}$to the origin and diagonalizes the linear part, reduces system (1.4) to

$$
\left\{\begin{array}{l}
\frac{d x}{d t}=-w y+m a_{1} x^{2}+2 a_{1} w x y-m a_{1}^{2} x^{3}-a_{1}^{2} w x^{2} y \\
\frac{d y}{d t}=w x-a_{1} b_{2} x^{2}-2 a_{1} x y+a_{1}^{2} b_{1} x^{3}+a_{1}^{2} x^{2} y
\end{array}\right.
$$

where

$$
\begin{aligned}
w & =\sqrt{k_{1}}, \quad k_{1}=-\frac{(2 m p+1)(2 m p-m q+1+q)}{(m p+p+1)^{2}}, \quad a_{1}=\frac{m p+p+1}{\sqrt{(m-1)(m p+p+1)}}, \\
b_{1} & =\frac{2 m^{3} p^{2}+p q m^{2}+3 m^{2} p+2 m p^{2}-2 p q m+m+p+q p}{(m p+p+1)^{2} w}, \\
b_{2} & =\frac{2 m^{3} p^{2}-2 m^{2} p^{2}+3 m^{2} p+2 q m^{2} p+4 m p^{2}-4 p q m-m p+m+2 q p+2 p}{(m p+p+1)^{2} w} .
\end{aligned}
$$

Obviously, $k_{1}>0$ because $m>1$ and $q>(2 m p+1) /(m-1)$. Using the polar 
coordinates $x_{1}=r \cos \theta, y_{1}=r \sin \theta$, we obtain from (3.1) that

$$
\begin{aligned}
\frac{d r}{d \theta}= & \frac{G_{2}(\theta)}{w} r^{2}+\left(\frac{G_{3}(\theta)}{w}-\frac{G_{2}(\theta) H_{1}(\theta)}{w^{2}}\right) r^{3}+\left(-\frac{G_{3}(\theta) H_{1}(\theta)}{w^{2}}-\frac{G_{2}(\theta) H_{2}(\theta)}{w^{2}}+\frac{G_{2}(\theta) H_{1}^{2}(\theta)}{w^{3}}\right) r^{4} \\
& +\left(-\frac{G_{3}(\theta) H_{2}(\theta)}{w^{2}}+\frac{2 G_{2}(\theta) H_{1}(\theta) H_{2}(\theta)}{w^{3}}+\frac{G_{3}(\theta) H_{1}^{2}(\theta)}{w^{3}}-\frac{G_{2}(\theta) H_{1}^{3}(\theta)}{w^{4}}\right) r^{5}+\text { h.o.t., }
\end{aligned}
$$

where

$$
\begin{aligned}
& G_{2}(\theta)=a_{1}(m+2) \sin ^{3} \theta+a_{1}\left(2 w-b_{2}\right) \sin ^{2} \theta \cos \theta-2 a_{1} \sin \theta, \\
& G_{3}(\theta)=-a_{1}^{2}(m+1) \sin ^{4} \theta+a_{1}^{2}\left(b_{1}-w\right) \sin ^{3} \theta \cos \theta+a_{1}^{2} \sin ^{2} \theta, \\
& H_{1}(\theta)=a_{1}\left(2 w-b_{2}\right) \sin ^{3} \theta-a_{1}(m+2) \sin ^{2} \theta \cos \theta-2 a_{1} w \sin \theta, \\
& H_{2}(\theta)=a_{1}^{2}\left(b_{1}-w\right) \sin ^{4} \theta+a_{1}^{2}(m+1) \sin ^{3} \theta \cos \theta+a_{1}^{2} w \sin ^{2} \theta,
\end{aligned}
$$

and

$$
G_{4}(\theta)=G_{5}(\theta)=H_{3}(\theta)=H_{4}(\theta)=H_{5}(\theta)=0 .
$$

Consider solutions of (3) in the formal series $r\left(\theta, r_{0}\right)=\sum_{j=1}^{+\infty} r_{j}(\theta) r_{0}^{j}$ together with the initial condition $r\left(0, r_{0}\right)=r_{0}$, where $\left|r_{0}\right|$ is sufficiently small. Obviously, $r_{1}(0)=$ $1, r_{2}(0)=r_{3}(0)=\cdots=0$. Substituting the series into (3) and comparing the coefficients, we obtain a system of differential equations for $r_{j}(\theta), j=1,2, \ldots$, i.e.,

$$
\frac{d r_{1}}{d \theta}=0, \quad \frac{d r_{2}}{d \theta}=r_{1}^{2} \frac{G_{2}(\theta)}{w}, \quad \frac{d r_{3}}{d \theta}=r_{1}^{3}\left(\frac{G_{3}(\theta)}{w}-\frac{G_{2}(\theta) H_{1}(\theta)}{w^{2}}\right)+2 r_{1} r_{2} \frac{G_{2}(\theta)}{w}, \ldots
$$

Solving them together with the initial conditions, we get

$$
\begin{aligned}
r_{1}(\theta) \equiv 1, \quad r_{2}(\theta) & =\int_{0}^{\theta} \frac{G_{2}(\xi)}{w} d \xi \\
r_{3}(\theta) & =\int_{0}^{\theta}\left\{\frac{G_{3}(\xi)}{w}-\frac{G_{2}(\xi) H_{1}(\xi)}{w^{2}}+\frac{2 G_{2}(\xi) r_{2}(\xi)}{w}\right\} d \xi, \ldots
\end{aligned}
$$

Using Maple V.7 software, we compute the Liapunov value as follows:

$$
L_{3}\left(m, w, a_{1}, b_{1}, b_{2}\right)=\frac{1}{2 \pi} r_{3}(2 \pi)=\frac{a_{1}^{2}(m-1)\left(2 b_{2}-w\right)}{8 w^{2}} .
$$

For parameters in $\Omega$, the sign of $L_{3}$ is determined by $2 b_{2}-w$ and therefore is the same as the sign of $\mu$, which demonstrates the corresponding results in Lemma 2.2.

If $w=2 b_{2}$ (i.e., $\left.\mu=0\right)$, then $L_{3}\left(m, w, a_{1}, b_{1}, b_{2}\right)=0$. In this case the Liapunov value of order 5 can be calculated as follows:

$$
L_{5}\left(m, w, a_{1}, b_{1}, b_{2}\right)=\frac{1}{2 \pi} r_{5}(2 \pi)=\frac{a_{1}^{4}(m-1) \kappa\left(m, w, a_{1}, b_{1}, b_{2}\right)}{768 w^{4}}>0,
$$

where, using (3.1) and the fact that $\mu=0$, we have

$$
\begin{aligned}
\kappa\left(m, w, a_{1}, b_{1}, b_{2}\right)= & 121 w^{3}-332 w^{2} b_{2}+143 w^{2} b_{1}-75 w m^{2}-218 w m-190 w b_{2} b_{1} \\
& -8 w+155 w b_{2}^{2}+150 b_{2} m^{2}+436 m b_{2}+16 b_{2}+50 b_{2}^{3} \\
= & \frac{(2 p+1)(m p+p+1)^{3}(-4 m p+8 p+2)^{1 / 2}}{64(m-1)(2 m p+1)^{2}\left(m^{2} p+m p+m\right)^{1 / 2}}>0 .
\end{aligned}
$$

Copyright $@$ by SIAM. Unauthorized reproduction of this article is prohibited. 
By the theory of Hopf bifurcation [20,32], we obtain the following results.

THEOREM 3.1. Suppose that $A^{2}>4 m(m p+q+1)$ and conditions in $\Omega$ hold.

(i) If $\mu \neq 0$, then the equilibrium $E_{+}$of system (1.4) is a weak focus of multiplicity 1 and at most one limit cycle arises from the Hopf bifurcation. Moreover, $E_{+}$is stable and the limit cycle is also stable when $\mu<0$, or $E_{+}$is unstable and the limit cycle is also unstable when $\mu>0$.

(ii) If $\mu=0$, then the equilibrium $E_{+}$is a weak focus of multiplicity 2 and at most two limit cycles arise from the Hopf bifurcation. Moreover, $E_{+}$is unstable and the outer cycle is also unstable, but the inner cycle (if it appears) is stable.

To carry out numerical simulations on two limit cycles, we choose parameters $m=3, p=0.1, q=15$, and $A=21.99$. We can verify that the conditions of result (ii) in Theorem 3.1 are satisfied. In this case the two limit cycles can be simulated by using MATLAB 6.5 software. In Figure 2, the trajectory started at the point $P_{1}=(1,5)$ spirals inward as the time $t \rightarrow-\infty$ and the trajectory started at $P_{2}=(1.2,12)$ spirals inward as $t \rightarrow \infty$. Hence, an unstable outer limit cycle exists and lies in the annular region bounded by these two trajectories. Similarly, the orbit started at $P_{3}=(1.5,15)$ spirals inward as $t \rightarrow \infty$ and the orbit started at $P_{4}=(1.3,17)$ spirals outward as $t \rightarrow \infty$. Therefore, there is a stable inner limit cycle lying in the annular region bounded by these two orbits. The equilibrium $E_{+}=(1.195073225,17.92609838)$ is unstable.

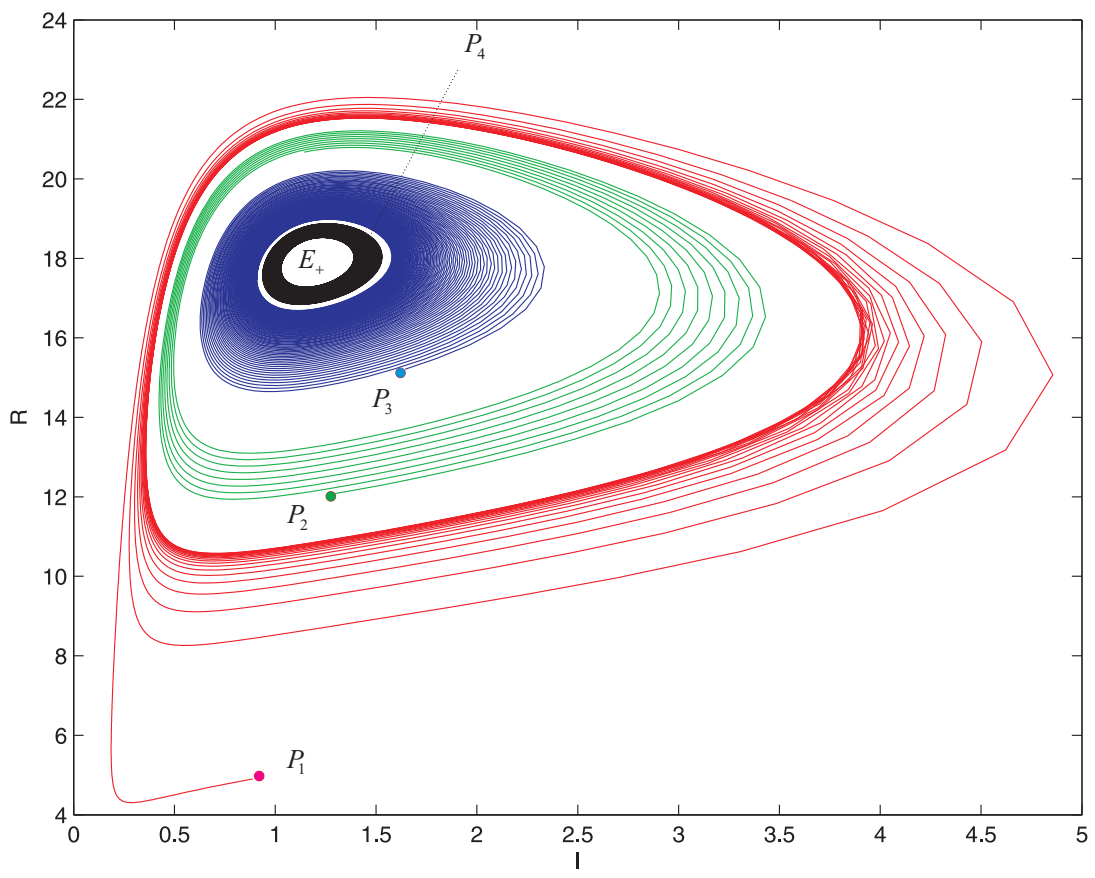

FIG. 2. Two limit cycles bifurcate from the weak focus of multiplicity 2.

4. Uniqueness of limit cycles. In this section we consider the uniqueness of limit cycles of system (1.4) and provide a relatively simpler proof compared to that of [25]. As shown in Theorem 2.2 of [25] and section 2, it suffices to discuss the case when $m>1$ and $A^{2}>4 m(m p+q+1)$, in which system (1.4) possibly has closed 
orbits and $E_{+}$lies in the first quadrant.

Our strategy is to reduce system (1.4) to the form of the Liénard system

$$
\dot{x}=y-F(x), \quad \dot{y}=-g(x)
$$

and apply the known Theorem 1.1 in Kooij and Zegeling [15] and Theorem 2.1 in Xiao and Zhang [29]. Rearranging terms in the order of powers of $R$, we rewrite system (1.4) as

$$
\dot{I}=g_{0}(I)-g_{1}(I) R, \quad \dot{R}=q I-R,
$$

where $g_{0}(I)=(A-I) I^{2} /\left(1+p I^{2}\right)-m I$ and $g_{1}(I)=I^{2} /\left(1+p I^{2}\right)$. We need only to consider $I>0$ because $I=0$ is an orbit. Thus $g_{1}(I)>0$, and system (4.2) has the same phase portrait as the system

$$
\dot{I}=R-\frac{g_{0}(I)}{g_{1}(I)}, \quad \dot{R}=\frac{R}{g_{1}(I)}-\frac{q I}{g_{1}(I)} .
$$

With the transformation

$$
I=x, \quad y=R-\int_{I_{+}}^{x} \frac{1}{g_{1}(x)} d x
$$

system (4.3) is reduced to the Liénard system (4.1) with

$$
F(x)=\frac{g_{0}(x)}{g_{1}(x)}-\int_{I_{+}}^{x} \frac{1}{g_{1}(x)} d x, \quad g(x)=\frac{q x}{g_{1}(x)}-\frac{g_{0}(x)}{g_{1}^{2}(x)} .
$$

Lemma 4.1. Suppose that $m>1$ and $A^{2}>4 m(m p+q+1)$. Then system (4.2) has at most one closed orbit in the interior of the first quadrant if either the function $F^{\prime}(x) / g(x)$, where $F^{\prime}$ denotes the derivative of $F$, is neither decreasing nor a constant or $F^{\prime}\left(I_{+}\right) \frac{d}{d x}\left(F^{\prime}(x) / g(x)\right)<0$ for $x \neq I_{+}$. Moreover, the closed orbit is hyperbolic if it exists.

Proof. Note that limit cycles of system (4.2) (if any exist) lie in the stripe region between the vertical lines $\ell_{0}: I=I_{-}$and $\ell_{1}: I=I_{D}$, where

$$
I_{D}=\frac{A+\left(A^{2}-4 m(m p+1)\right)^{1 / 2}}{2(m p+1)} .
$$

In fact, a vertical isocline of (4.2) intersects the $I$-axis at $D=\left(I_{D}, 0\right)$, i.e., $(m p+$ 1) $I_{D}^{2}-A I_{D}+m=0$. Restricted to $\ell_{1}$ in the interior of the first quadrant, the derivative $\dot{I}$ satisfies

$$
\left.\dot{I}\right|_{\ell_{1}}=I_{D}\left\{\left[-m+A I_{D}-(m p+1) I_{D}^{2}\right]-R I_{D}\right\}=-R I_{D}^{2}<0 .
$$

This implies that limit cycles of system (4.2) (if any exist) lie on the left of $\ell_{1}$ because $I_{+}<I_{D}$ (i.e., the equilibrium $E_{+}$lies on the left of $\ell_{1}$ ). On the other hand, limit cycles lie on the right of $\ell_{0}$; otherwise, a limit cycle intersects $\ell_{0}$ because $I_{+}>I_{-}$ (i.e., $E_{+}$lies on the right of $\ell_{0}$ ), implying that $\dot{I}=0$ at a point on $\ell_{0}$. This is a contradiction because on $\ell_{0}$ the derivative

$$
\dot{I}=I_{-}\left\{\left[-m+\left(A-R_{-}\right) I_{-}-(m p+1) I_{-}^{2}\right]+R_{-} I_{-}-R I_{-}\right\}=\left(R_{-}-R\right) I_{-}^{2} \neq 0
$$


except at the saddle $E_{-}$. Let $S\left(I_{-}, I_{D}\right)$ denote the stripe region. Since transformations between (4.2) and (4.1) do not change $x$, it suffices to discuss (4.1) in $S\left(I_{-}, I_{D}\right)$, i.e., $I_{-}<x<I_{D}$.

Transformation (4.4) is one-to-one for $I>0$ and $R>0$, so it is equivalent to discuss the uniqueness of closed orbits for system (4.1) where $x>0$. Corresponding to $E_{+}$, system (4.1) has an equilibrium $\left(x_{+}, y_{+}\right)$with the same coordinates, i.e., $x_{+}=I_{+}$and $y_{+}=R_{+}$. From (4.5) we have $F^{\prime}(x)=-\left\{(m p+p+1) x^{2}+1-m\right\} / x^{2}$ and $g(x)=\rho(x)\left(1+p x^{2}\right) / x^{3}$, where $\rho(x)=(m p+q+1) x^{2}-A x+m$ has exactly two zeros at $I_{-}$and $I_{+}$. It follows that

$$
g\left(x_{+}\right)=-\frac{g_{0}\left(I_{+}\right)-g_{1}\left(I_{+}\right) R_{+}}{g_{1}^{2}\left(I_{+}\right)}=0, \quad\left(x-x_{+}\right) g(x)=\left(\frac{1+p x^{2}}{x^{3}}\right)\left(x-x_{+}\right) \rho(x)>0
$$

in $S\left(I_{-}, I_{D}\right)$, verifying partly either the condition in [29, Theorem 2.1] (as $F^{\prime}(x) / g(x)$ is neither decreasing nor a constant) or the condition in [15, Theorem 1.1] (as $F^{\prime}\left(I_{+}\right)$ $\cdot(d / d x)\left\{F^{\prime}(x) / g(x)\right\}<0$ for $\left.x \neq I_{+}\right)$. The other conditions can be checked explicitly by the assumptions in our lemma. Thus the lemma is proved.

In order to obtain conditions in terms of the original parameters for the uniqueness of limit cycles and complete the results of the uniqueness in [25], we use the notation

$c_{0}=m(m-1), \quad c_{2}=5 m^{2} p+m p+4 m+m q-q-1, \quad c_{3}=2 A(1+2 m p)$,

$c_{4}=\left(5 m^{2} p^{2}-m p^{2}\right)+(4 m p q-2 p q)+(6 m p-2 p)+q+1, \quad c_{6}=p(1+p+m p)(1+q+m p)$.

These constants are obviously all positive. Using the conditions in Lemma 4.1, we obtain the following theorem.

Theorem 4.2. Suppose that $m>1$ and $A^{2}>4 m(m p+q+1)$. If either (i) $c_{3}^{2}-4 c_{2} c_{4}>0$ and $\sigma_{-}<I_{-}, \sigma_{+}>I_{D}$, where $\sigma_{ \pm}=\left(c_{3} \pm \sqrt{c_{3}^{2}-4 c_{2} c_{4}} /\left(2 c_{4}\right)\right.$, or (ii) $c_{3}^{2}-4 c_{2} c_{4}<0, F^{\prime}\left(I_{+}\right)>0$, and both $c_{6}$ and $m(m-1)$ are small enough, then system (4.2) has at most one limit cycle in the interior of the first quadrant. Moreover, under assumption (i) (resp., (ii)) the limit cycle is unstable (resp., stable) if it exists.

Proof. Calculate $(d / d x)\left\{F^{\prime}(x) / g(x)\right\}=h(x) / x^{6} g^{2}(x)$ in $S\left(I_{-}, I_{D}\right)$, where

$$
h(x)=c_{0}-c_{2} x^{2}+c_{3} x^{3}-c_{4} x^{4}+c_{6} x^{6} .
$$

By Lemma 4.1, we need to determine the sign of $h(x)$. Our strategy is to discuss the quadratic function $\eta(x)=-c_{2}+c_{3} x-c_{4} x^{2}$, for which we have $h(x)=m(m-1)+$ $c_{6} x^{6}+\eta(x) x^{2}$.

In case (i), the function $\eta$ has exactly two real roots $\sigma_{ \pm}$and $\left[I_{-}, I_{D}\right] \subset\left(\sigma_{-}, \sigma_{+}\right)$. Since $\eta$ has the leading coefficient $c_{4}>0$, we see that $\eta(x)>0$ for $x \in\left(\sigma_{-}, \sigma_{+}\right)$, i.e., $\eta(x)>0$ in $S\left(I_{-}, I_{D}\right)$. Since $m>1$, as required in $(2.1)$, we have $h(x)>0$ or equivalently $(d / d x)\left\{F^{\prime}(x) / g(x)\right\}>0$ in $S\left(I_{-}, I_{D}\right)$. Then Lemma 4.1 implies the result in case (i).

In case (ii), $c_{3}^{2}-4 c_{2} c_{4}<0$, so $\eta(x) \neq 0$. On the compact interval $\left[I_{-}, I_{D}\right]$ the function $|\eta|$ is bounded by a positive number. Therefore, $\operatorname{sgnh}(x)=\operatorname{sgn} \eta(x)$ for $x \in\left(I_{-}, I_{D}\right)$ as $c_{6}$ and $m(m-1)$ are both sufficiently small. Since $\eta$ has the leading coefficient $c_{4}>0$, we ensure that $\eta(x)<0$ as $x \in\left(I_{-}, I_{D}\right)$, implying that $h(x)<0$ in $S\left(I_{-}, I_{D}\right)$. Thus, $F^{\prime}\left(I_{+}\right)(d / d x)\left\{F^{\prime}(x) / g(x)\right\}<0$; i.e., the second condition in Lemma 4.1 holds in $S\left(I_{-}, I_{D}\right)$. From the notion introduced at the beginning of the proof of Lemma 4.1, it suffices to verify the condition in $S\left(I_{-}, I_{D}\right)$. Therefore, the conclusion in case (ii) is obtained. 
Consider $p=q=\epsilon^{k}, m=1 / \epsilon$, and $(2 A(2 m p+1))^{2}=a_{1} c_{2} c_{4}$, for example, where $\epsilon>0$ is sufficiently small, $k \geq 3$, and $4<a_{1}<9 / 2$. These parameters satisfy assumption (i) in Theorem 4.2. Another choice of parameters in which $p=\epsilon^{2 k}$, $m=1+\epsilon$, and $q=1 / \epsilon^{k}$, where $\epsilon>0$ is sufficiently small and $k \geq 3$, verifies assumption (ii) in Theorem 4.2.

For $c_{6}$ and $m(m-1)$ to be sufficiently small in case (ii) of Theorem 4.2 , we have to restrict $p$ and $m$ near 0 and 1, respectively. Efforts are also made to extend the restriction by some known results on the zeros of high degree polynomials (Yang [31]). As shown in the above proof for Theorem 4.2 (ii), we can generally suppose that $m>1, A^{2}>4 m(m p+q+1)$ and that $h\left(I_{-}\right)<0, h\left(I_{D}\right)<0$ and claim that $h$ has no real zeros in the interval $\left(I_{-}, I_{D}\right)$. By Lemma 3.1 in [31], the number of real zeros of $h$ in $\left(I_{-}, I_{D}\right)$ is equal to the number of negative zeros of the function

$$
\Psi(x)=(1-x)^{6} h\left(\frac{I_{D}-I_{-} x}{1-x}\right)=\alpha_{0} x^{6}+\alpha_{1} x^{5}+\alpha_{2} x^{4}+\alpha_{3} x^{3}+\alpha_{4} x^{2}+\alpha_{5} x+\alpha_{6},
$$

where

$$
\begin{aligned}
\alpha_{0}= & h\left(I_{-}\right)=c_{0}-c_{2} I_{-}^{2}+c_{3} I_{-}^{3}-c_{4} I_{-}^{4}+c_{6} I_{-}^{6}, \\
\alpha_{1}= & -3 c_{3} I_{D} I_{-}^{2}+4 c_{4} I_{D} I_{-}^{3}-6 c_{6} I_{D} I_{-}^{5}+2 c_{2} I_{D} I_{-}+2 c_{4} I_{-}^{4}-6 c_{0}+4 c_{2} I_{-}^{2}-3 c_{3} I_{-}^{3}, \\
\alpha_{2}= & 9 c_{3} I_{D} I_{-}^{2}+3 c_{3} I_{D}^{2} I_{-}-8 c_{4} I_{D} I_{-}^{3}-6 c_{4} I_{D}^{2} I_{-}^{2}+15 c_{6} I_{D}^{2} I_{-}^{4}-8 c_{2} I_{D} I_{-}-c_{4} I_{-}^{4} \\
& +15 c_{0}-c_{2} I_{D}^{2}-6 c_{2} I_{-}^{2}+3 c_{3} I_{-}^{3}, \\
\alpha_{3}= & -9 c_{3} I_{D} I_{-}^{2}-9 c_{3} I_{D}^{2} I_{-}+4 c_{4} I_{D} I_{-}^{3}+12 c_{4} I_{D}^{2} I_{-}^{2}+4 c_{4} I_{D}^{3} I_{-} \\
& -20 c_{6} I_{D}^{3} I_{-}^{3}+12 c_{2} I_{D} I_{-}-20 c_{0}+4 c_{2} I_{D}^{2}+4 c_{2} I_{-}^{2}-c_{3} I_{-}^{3}-c_{3} I_{D}^{3}, \\
\alpha_{4}= & 3 c_{3} I_{D} I_{-}^{2}+9 c_{3} I_{D}^{2} I_{-}-6 c_{4} I_{D}^{2} I_{-}^{2}-8 c_{4} I_{D}^{3} I_{-}+15 c_{6} I_{D}^{4} I_{-}^{2}-8 c_{2} I_{D} I_{-}+15 c_{0} \\
& -c_{4} I_{D}^{4}-6 c_{2} I_{D}^{2}-c_{2} I_{-}^{2}+3 c_{3} I_{D}^{3}, \\
\alpha_{5}= & -3 c_{3} I_{D}^{3}-3 c_{3} I_{D}^{2} I_{-}-6 c_{6} I_{D}^{5} I_{-}+4 c_{2} I_{D}^{2}+2 c_{2} I_{D} I_{-}+2 c_{4} I_{D}^{4}+4 c_{4} I_{D}^{3} I_{-}-6 c_{0}, \\
\alpha_{6}= & h\left(I_{D}\right)=c_{0}-c_{2} I_{D}^{2}+c_{3} I_{D}^{3}-c_{4} I_{D}^{4}+c_{6} I_{D}^{6} .
\end{aligned}
$$

Let $\operatorname{Discr}(\Psi)$ be the discrimination matrix of the polynomial $\Psi$, constructed in the appendix as in [31, Definition 2.1] and its following paragraph, and calculate its principal minors $\varpi_{1}, \varpi_{2}, \ldots, \varpi_{13}$ as in the appendix. Consider the sequence $S E=$ $\left\{\varpi_{1} \varpi_{2}, \varpi_{2} \varpi_{3}, \ldots, \varpi_{12} \varpi_{13}\right\}$ and its sign list $\mathcal{S}(S E)=\left\{\operatorname{sgn}\left(\varpi_{1} \varpi_{2}\right), \ldots, \operatorname{sgn}\left(\varpi_{12} \varpi_{13}\right)\right\}$, where $\operatorname{sgn}(x)$ denotes the sign of $x$. Now revise the signs according to the following rule (Definition 2.3 in [31]): (S1) If $\left\{\varrho_{i}, \varrho_{i+1}, \ldots, \varrho_{i+j}\right\}$ is a section of $\mathcal{S}(S E)$ such that $\varrho_{i} \neq 0, \varrho_{i+1}=\cdots=\varrho_{i+j-1}=0, \varrho_{i+j} \neq 0$, then replace the section with the finite sequence $\left\{-\varrho_{i},-\varrho_{i}, \varrho_{i}, \varrho_{i},-\varrho_{i},-\varrho_{i}, \varrho_{i}, \varrho_{i}, \ldots\right\}$ by truncating for the same number of terms; (S2) otherwise, do not change. Let $\mathcal{S}^{\prime}(S E)$ denote the revised sign list. By Theorem 3.3 in [31], the number of distinct negative zeros of $\Psi$ is equal to $\xi_{1}-\xi$, where $\xi$ is the number of sign changes in $\mathcal{S}^{\prime}(S E)$ and $2 \xi_{1}$ is the number of nonzero members in $\mathcal{S}^{\prime}(S E)$. Thus we conclude that $\Psi$ has no negative zeros, i.e., $h(x)<0$ in $\left(I_{-}, I_{D}\right)$ if $\xi=\xi_{1}$.

The above conclusion shows that the condition on parameters for $h(x)<0$ can be determined by the list $\mathcal{S}^{\prime}(S E)$. It is easy to calculate that $\varpi_{1} \varpi_{2}=6 \alpha_{0}^{3}<0$, i.e., $\operatorname{sgn}\left(\varpi_{1} \varpi_{2}\right)=-1$. So in total we have $3^{11}(=177147)$ cases to discuss because each of the remaining 11 elements in $\mathcal{S}^{\prime}(S E)$ has three options: $-1,0,1$. We illustrate a general method for conditions on parameters with a further discussion on $\varpi_{12} \varpi_{13}$. In the case that $\varpi_{12} \varpi_{13} \neq 0, \mathcal{S}^{\prime}(S E)$ contains 12 nonzero members, implying that $\xi_{1}=6$. So we need only to construct a revised sign list with $\xi=6$. We easily 
find such a list $\{-1,1,-1,1,-1,1,-1,-1,-1,-1,-1,-1\}$, which gives a condition on parameters:

$$
\begin{aligned}
\left(\mathrm{C}_{1}\right): \varpi_{2} \varpi_{3} & \geq 0, \varpi_{3} \varpi_{4}<0, \varpi_{4} \varpi_{5}>0, \varpi_{5} \varpi_{6}<0, \varpi_{6} \varpi_{7}>0, \varpi_{7} \varpi_{8}<0, \\
\varpi_{8} \varpi_{9} & <0, \varpi_{9} \varpi_{10}<0, \varpi_{10} \varpi_{11}<0, \varpi_{11} \varpi_{12}<0, \varpi_{12} \varpi_{13}<0 .
\end{aligned}
$$

In the case that $\varpi_{12} \varpi_{13}=0$, the number of nonzero members in $\mathcal{S}^{\prime}(S E)$ is $<12$, i.e., $\xi_{1} \leq 5$. Note that the list $\{-1,1,-1,1,-1,1,1,1,1,1,0,0\}$ has $\xi=5$. Being a revised sign list, it gives a condition of parameters:

$$
\begin{aligned}
\left(\mathrm{C}_{2}\right): \varpi_{2} \varpi_{3} & \geq 0, \varpi_{3} \varpi_{4}<0, \varpi_{4} \varpi_{5}>0, \varpi_{5} \varpi_{6}<0, \varpi_{6} \varpi_{7}>0, \varpi_{7} \varpi_{8}>0, \\
\varpi_{8} \varpi_{9} & >0, \varpi_{9} \varpi_{10}>0, \varpi_{10} \varpi_{11}>0, \varpi_{11} \varpi_{12}=\varpi_{12} \varpi_{13}=0 .
\end{aligned}
$$

Finally, Lemma 4.1 and the conclusion given in the last paragraph enable us to summarize that if $m>1, A^{2}>4 m(m p+q+1), h\left(I_{-}\right)<0, h\left(I_{D}\right)<0, F^{\prime}\left(I_{+}\right)>0$, and if either $\left(C_{1}\right)$ or $\left(C_{2}\right)$ holds, then system (4.2) has at most one limit cycle in the interior of the first quadrant. Moreover, the limit cycle is stable if it exists. More conditions other than $\left(C_{1}\right)$ and $\left(C_{2}\right)$ can similarly be obtained for the uniqueness of limit cycles.

5. Degenerate Bogdanov-Takens bifurcation. By Lemma 2.3, when $A=$ $A_{0}=: 2 \sqrt{m(m p+q+1)}$ and $p=p_{0}=:((m-1) q-1) /(2 m)$, the equilibrium $E_{0}=$ : $\left(A_{0} /\left(2\left(m p_{0}+q+1\right)\right), q A_{0} /\left(2\left(m p_{0}+q+1\right)\right)\right)$ is a cusp, where the Bogdanov-Takens bifurcation may occur by a perturbation. By the standard theory of the BogdanovTakens bifurcation (of codimension 2), Ruan and Wang [25] assert only that the system has at most one limit cycle and the obtained homoclinic loop is of order 1 (see the definition in [16]).

In the following, we display the possible bifurcations of multiple limit cycles and homoclinic loops of order higher than 1 . Note that as $A=A_{0}$ and $p=p_{0}$, we have $(m-1) q=2 m p_{0}+1>0$, implying $m>1$. So we fix $m_{0}>1$ near 1 arbitrarily and consider three bifurcation parameters $A, p, m$ near $A_{0}, p_{0}, m_{0}$, respectively. Let

$$
A=A_{0}+\epsilon_{1}, \quad p=p_{0}+\epsilon_{2}, \quad m=m_{0}+\epsilon_{3},
$$

where $\epsilon_{3}>0$. Then, we discuss bifurcations of the equivalent system (1.4) for the parameters $\epsilon=\left(\epsilon_{1}, \epsilon_{2}, \epsilon_{3}\right)$ near $(0,0,0)$.

Lemma 5.1. For $A, p, m$ close to $A_{0}, p_{0}, m_{0}$, respectively, (1.4) is equivalent to the system

$$
\frac{d x}{d t}=y, \quad \frac{d y}{d t}=\mu_{1}+x^{2}+\left(\mu_{2}+\mu_{3} x+x^{3}+O\left(|x|^{4}\right)\right) y+G(x, \mu) y^{2},
$$

where $\mu_{i}$ 's are functions of $\epsilon_{1}, \epsilon_{2}, \epsilon_{3}$ such that $\left.\frac{\partial\left(\mu_{1}, \mu_{2}, \mu_{3}\right)}{\partial\left(\epsilon_{1}, \epsilon_{2}, \epsilon_{3}\right)}\right|_{\epsilon=0} \neq 0$ and $G(x, \mu)$ is a $C^{\infty}$ function.

Proof. With the substitution (5.1), equation (1.4) can be written as

$$
\left\{\begin{array}{l}
\dot{I}=\mathcal{I}\left(I+\frac{A_{0}}{2\left(\left(m_{0}+\epsilon_{3}\right)\left(p_{0}+\epsilon_{2}\right)+q+1\right)}, R+\frac{q A_{0}}{2\left(\left(m_{0}+\epsilon_{3}\right)\left(p_{0}+\epsilon_{2}\right)+q+1\right)}\right), \\
\dot{R}=\mathcal{R}\left(I+\frac{A_{0}}{2\left(\left(m_{0}+\epsilon_{3}\right)\left(p_{0}+\epsilon_{2}\right)+q+1\right)}, R+\frac{q A_{0}}{2\left(\left(m_{0}+\epsilon_{3}\right)\left(p_{0}+\epsilon_{2}\right)+q+1\right)}\right)
\end{array}\right.
$$

where $\mathcal{I}$ and $\mathcal{R}$ are defined in (1.4). When $\epsilon=0$, system (5.3) has a cusp at the origin $\mathrm{O}_{2}=(0,0)$, as shown in [25]; i.e., the equilibrium $E_{0}$ is translated to $\mathrm{O}_{2}$. Expanding (5.3) at $O_{2}$, rescaling time by $t=\tau\left(q+1+q m_{0}\right) /\left(2 q m_{0}\right)$, and then applying a linear 
transformation $T_{1}:(I, R) \mapsto(\tilde{I}, \tilde{R})$, defined by $\tilde{I}=I$ and $\tilde{R}=I-R / q$ to reduce the matrix of the linear part for $\epsilon=0$ to the Jordan canonical form, we can reduce (5.3) further to the form

$$
\left[\begin{array}{c}
\dot{\tilde{I}} \\
\tilde{\tilde{R}}
\end{array}\right]=\left[\begin{array}{l}
\vartheta_{1}(\epsilon) \\
\vartheta_{2}(\epsilon)
\end{array}\right]+\left[\begin{array}{ll}
\iota_{11}(\epsilon) & \iota_{12}(\epsilon) \\
\iota_{21}(\epsilon) & \iota_{22}(\epsilon)
\end{array}\right]\left[\begin{array}{c}
\tilde{I} \\
\tilde{R}
\end{array}\right]+\left[\begin{array}{l}
\omega_{1}(\tilde{I}, \tilde{R}, \epsilon) \\
\omega_{2}(\tilde{I}, \tilde{R}, \epsilon)
\end{array}\right],
$$

where all $\vartheta_{j}, \iota_{i j}$, and $\omega_{j}(i, j=1,2)$ are calculated as in the appendix, which satisfies that $\vartheta_{1}(0)=\vartheta_{2}(0)=0, \iota_{12}(0)=1$, and $\iota_{11}(0)=\iota_{21}(0)=\iota_{22}(0)=0$. Another transformation $T_{2}:(\tilde{I}, \tilde{R}) \mapsto(X, Y)$, defined by

$$
\tilde{I}=X+\frac{\sqrt{2 m_{0}\left(q+1+q m_{0}\right)}}{2 m_{0}} X^{2}, \quad \tilde{R}=Y+\frac{\sqrt{2 m_{0}\left(q+1+q m_{0}\right)}\left(q+1+q m_{0}\right)}{4 q m_{0}} X^{2},
$$

reduces system (5.4) to

$$
\dot{X}=E_{11}(X, \epsilon)+E_{12}(X, \epsilon) Y, \quad \dot{Y}=E_{21}(X, \epsilon)+E_{22}(X, \epsilon) Y,
$$

where

$$
\begin{array}{ll}
E_{1 j}(X, \epsilon)=a_{j 0}(\epsilon)+a_{j 1}(\epsilon) X+a_{j 2}(\epsilon) X^{2}+a_{j 3}(\epsilon) X^{3}+O\left(\left|X^{4}\right|\right), & j=1,2, \\
E_{2 j}(X, \epsilon)=b_{j 0}(\epsilon)+b_{j 1}(\epsilon) X+b_{j 2}(\epsilon) X^{2}+b_{j 3}(\epsilon) X^{3}+O\left(\left|X^{4}\right|\right), & j=1,2,
\end{array}
$$

and all $a_{i j}(\epsilon)$ 's and $b_{i j}(\epsilon)$ 's are given in the appendix. Applying the change of variables $\tilde{X}=X, \tilde{Y}=E_{11}(X, \epsilon)+E_{12}(X, \epsilon) Y$ in (5.6), we obtain a system in which the first equation is same as the first equation of (5.2), that is,

$$
\dot{\tilde{X}}=\tilde{Y}, \quad \dot{\tilde{Y}}=F_{1}(\tilde{X}, \epsilon)+F_{2}(\tilde{X}, \epsilon) \tilde{Y}+F_{3}(\tilde{X}, \epsilon) \tilde{Y}^{2},
$$

where $F_{i}(\tilde{X}, \epsilon)=\sum_{j=0}^{3} c_{i j}(\epsilon) \tilde{X}^{j}+O\left(|\tilde{X}|^{4}\right), i=1,2$, and both $c_{i j}(\epsilon)^{\prime}$ 's and $F_{3}(\tilde{X}, \epsilon)$ are given in the appendix. Note that $c_{12}(0)=-\sqrt{2 m_{0}\left(q+1+q m_{0}\right)}\left(q+1+q m_{0}\right) / 4 q m_{0}$ $<0$, which implies that $c_{12}(\epsilon)<0$ for small $\epsilon$, and it is reasonable to apply the rescaling $\tilde{X} \mapsto-\tilde{X}, \tilde{Y} \mapsto-\sqrt{-c_{12}(\epsilon)} \tilde{Y}, \tau \mapsto \tau / \sqrt{-c_{12}(\epsilon)}$ to system (5.7) and obtain

$$
\dot{\tilde{X}}=\tilde{Y}, \quad \dot{\tilde{Y}}=\tilde{F}_{1}(\tilde{X}, \epsilon)+\tilde{F}_{2}(\tilde{X}, \epsilon) \tilde{Y}+\tilde{F}_{3}(\tilde{X}, \epsilon) \tilde{Y}^{2},
$$

where $\tilde{F}_{1}(\tilde{X}, \epsilon)=c_{10}(\epsilon) / c_{12}(\epsilon)-\left(c_{11}(\epsilon) / c_{12}(\epsilon)\right) \tilde{X}+\tilde{X}^{2}+O\left(|\tilde{X}|^{3}\right), \tilde{F}_{2}(\tilde{X}, \epsilon)=\left\{c_{20}(\epsilon)-\right.$ $\left.c_{21}(\epsilon) \tilde{X}+c_{22}(\epsilon) \tilde{X}^{2}-c_{23}(\epsilon) \tilde{X}^{3}\right\} / \sqrt{-c_{12}(\epsilon)}+O\left(|\tilde{X}|^{4}\right)$, and $\tilde{F}_{3}(\tilde{X}, \epsilon)=-F_{3}(-\tilde{X}, \epsilon)$. Thus, the coefficient of $\tilde{X}^{2}$ in $\tilde{F}_{1}$ in the second equation of (5.8) reduces to 1 , the same as the corresponding one in (5.2).

In order to reduce system (5.8) to the induced form (5.2), we need to remove the term of $\tilde{X}$ in the second equation of (5.8). We achieve this by the affine transformation $u=\tilde{X}-c_{11}(\epsilon) / 2 c_{12}(\epsilon), v=\tilde{Y}$ in $\tilde{X}$, and change system (5.8) into

$$
\dot{u}=v, \quad \dot{v}=G_{1}(u, \epsilon)+G_{2}(u, \epsilon) v+G_{3}(u, \epsilon) v^{2},
$$

where $G_{1}(u, \epsilon)=d_{10}(\epsilon)+u^{2}+O\left(|u|^{3}, \epsilon^{2}\right), G_{2}(u, \epsilon)=d_{20}(\epsilon)+d_{21}(\epsilon) u+d_{22}(\epsilon) u^{2}+$ $d_{23}(\epsilon) u^{3}+O\left(|u|^{4}\right), G_{3}(u, \epsilon)=-F_{3}\left(-u-c_{11} /\left(2 c_{12}\right), \epsilon\right)$, and the coefficients $d_{i j}(\epsilon)$ are displayed in the appendix. Because

Copyright (c) by SIAM. Unauthorized reproduction of this article is prohibited. 


$$
d_{23}(0)=\frac{2^{-\frac{3}{4}}\left\{m_{0}\left(q+1+q m_{0}\right)\right\}^{1 / 4}\left(q+1+q m_{0}\right)^{1 / 2}\left(3 m_{0}-1\right)\left(q+1+q m_{0}\right)}{m_{0}^{2}\left(q m_{0}\right)^{1 / 2}}>0,
$$

for small $\epsilon \neq 0$ system $(5.9)$ can be rescaled by $\tilde{u}=d_{23}^{2 / 5}(\epsilon) u, \tilde{v}=d_{23}^{3 / 5}(\epsilon) v, \tilde{\tau}=$ $d_{23}^{-1 / 5}(\epsilon) \tau$ into the form

$$
\dot{\tilde{u}}=\tilde{v}, \quad \dot{\tilde{v}}=\tilde{G}_{1}(\tilde{u}, \epsilon)+\tilde{G}_{2}(\tilde{u}, \epsilon) \tilde{v}+\tilde{G}_{3}(\tilde{u}, \epsilon) \tilde{v}^{2},
$$

where $\tilde{G}_{1}(\tilde{u}, \epsilon)=d_{23}^{4 / 5} d_{10}(\epsilon)+\tilde{u}^{2}+O\left(|\tilde{u}|^{3}\right), \tilde{G}_{2}(\tilde{u}, \epsilon)=d_{23}^{1 / 5} d_{20}(\epsilon)+d_{23}^{-1 / 5} d_{21}(\epsilon) \tilde{u}+$ $d_{23}^{-3 / 5} d_{22}(\epsilon) \tilde{u}^{2}+\tilde{u}^{3}+O\left(|\tilde{u}|^{4}\right)$, and $\tilde{G}_{3}(\tilde{u}, \epsilon)=-d_{23}^{-2 / 5} F_{3}\left(-d_{23}^{-2 / 5} \tilde{u}-c_{11} /\left(2 c_{12}\right), \epsilon\right)$, so that the coefficient of the term $\tilde{u}^{3} \tilde{v}$ in the second equation of (5.10) becomes 1 , the same as in the corresponding term in system (5.2). The invertibility of all undergone transformations for small $\epsilon \neq 0$ implies that system (5.10) is topologically conjugate to system (5.2) locally. Hence (5.3) is an induced family of vector fields from system (5.2), the universal unfolding of the degenerate cusp as shown in the Main Theorem in [7]. Comparing the 4-jet of (5.10) with (5.2), we obtain the relation between the induced system and the universal unfolding, i.e.,

$$
\mu_{1}(\epsilon)=d_{23}^{4 / 5} d_{10}(\epsilon), \quad \mu_{2}(\epsilon)=d_{23}^{1 / 5} d_{20}(\epsilon), \quad \mu_{3}(\epsilon)=d_{23}^{-1 / 5} d_{21}(\epsilon) .
$$

In particular, $\mu_{1}(0)=\mu_{2}(0)=\mu_{3}(0)=0$. Computing the Jacobian determinant of relation (5.11) at $(0,0,0)$ with Maple V.7 software, we get

$$
\left.\frac{\partial\left(\mu_{1}, \mu_{2}, \mu_{3}\right)}{\partial\left(\epsilon_{1}, \epsilon_{2}, \epsilon_{3}\right)}\right|_{\epsilon=0}=\frac{\left.1+q+4 m_{0}+3 q m_{0}+5 q m_{0}^{2}-m_{0}^{2}-m_{0}^{3} q\right)\left(q+1+q m_{0}\right)^{2}}{2},
$$

which is $>0$ for $m_{0}$ near 1 . This implies that the induced family (5.3) parameterized by $\epsilon$, and therefore (1.4), is locally equivalent to the unfolding (5.2). The proof is completed.

Concerning the universal unfolding (5.2), Theorem 4 in [7] gives bifurcation surfaces

$$
\begin{aligned}
& \mathcal{S N}=\left\{\left(\mu_{1}, \mu_{2}, \mu_{3}\right) \in V \mid \mu_{1}=0\right\}, \\
& \mathcal{H}=\left\{\left(\mu_{1}, \mu_{2}, \mu_{3}\right) \in V \mid \mu_{2}=\mu_{3}\left(-\mu_{1}\right)^{\frac{1}{2}}+\left(-\mu_{1}\right)^{\frac{3}{2}}+O\left(\left(-\mu_{1}\right)^{\frac{7}{4}}\right), \mu_{1}<0\right\}, \\
& \mathcal{H} \mathcal{L}=:\left\{\left(\mu_{1}, \mu_{2}, \mu_{3}\right) \in V \mid \mu_{2}=\frac{5}{7} \mu_{3}\left(-\mu_{1}\right)^{\frac{1}{2}}+\frac{103}{77}\left(-\mu_{1}\right)^{\frac{3}{2}}+O\left(\left(-\mu_{1}\right)^{\frac{7}{4}}\right), \mu_{1}<0\right\}, \\
& \mathcal{L}=:\left\{\left(\mu_{1}, \mu_{2}, \mu_{3}\right) \in V \mid \Xi\left(\mu_{1}, \mu_{2}, \mu_{3}\right)=0, \mu_{1}<0\right\},
\end{aligned}
$$

where $V$ is a neighborhood of $(0,0,0)$ and the surface $\Xi\left(\mu_{1}, \mu_{2}, \mu_{3}\right)=0$ is defined by

$\mu_{2}=\left(-\mu_{1}\right)^{\frac{3}{2}}\left(-\frac{6 P(b)}{11 P^{\prime}(b)}+\frac{6 b}{11}\right)+o\left(\left(-\mu_{1}\right)^{\frac{3}{2}}\right), \quad \mu_{3}=-\mu_{1}\left(-\frac{6}{11 P^{\prime}(b)}-\frac{15}{11}\right)+o\left(-\mu_{1}\right)$

for $\mu_{1}<0$ with the parameter $b$. Here $P(b)$ is a solution of the Riccati equation $\left(9 b^{2}-4\right) P^{\prime}=7 P^{2}+3 b P-5$, as shown in [7]. Applying the inverse of (5.11) together with (5.1), from $\mathcal{S N}, \mathcal{H}, \mathcal{H} \mathcal{L}$, and $\mathcal{L}$ we can give for system (1.4) the corresponding bifurcation surfaces $\mathcal{S N}^{\prime}, \mathcal{H}^{\prime}, \mathcal{H} \mathcal{L}^{\prime}$, and $\mathcal{L}^{\prime}$, respectively. Thus, Theorem 4 in [7] implies the following.

THEOREM 5.2. In the $(A, p, m)$-space there are four surfaces $\mathcal{S N}^{\prime}, \mathcal{H}^{\prime}, \mathcal{H} \mathcal{L}^{\prime}, \mathcal{L}^{\prime}$ near $\left(A_{0}, p_{0}, m_{0}\right)$, defined as above, such that system (1.4) produces a saddle-node bifurcation near $E_{0}$ as $(A, p, m)$ crosses $\mathcal{S N}^{\prime}$, a Hopf bifurcation near $E_{0}$ as $(A, p, m)$ 


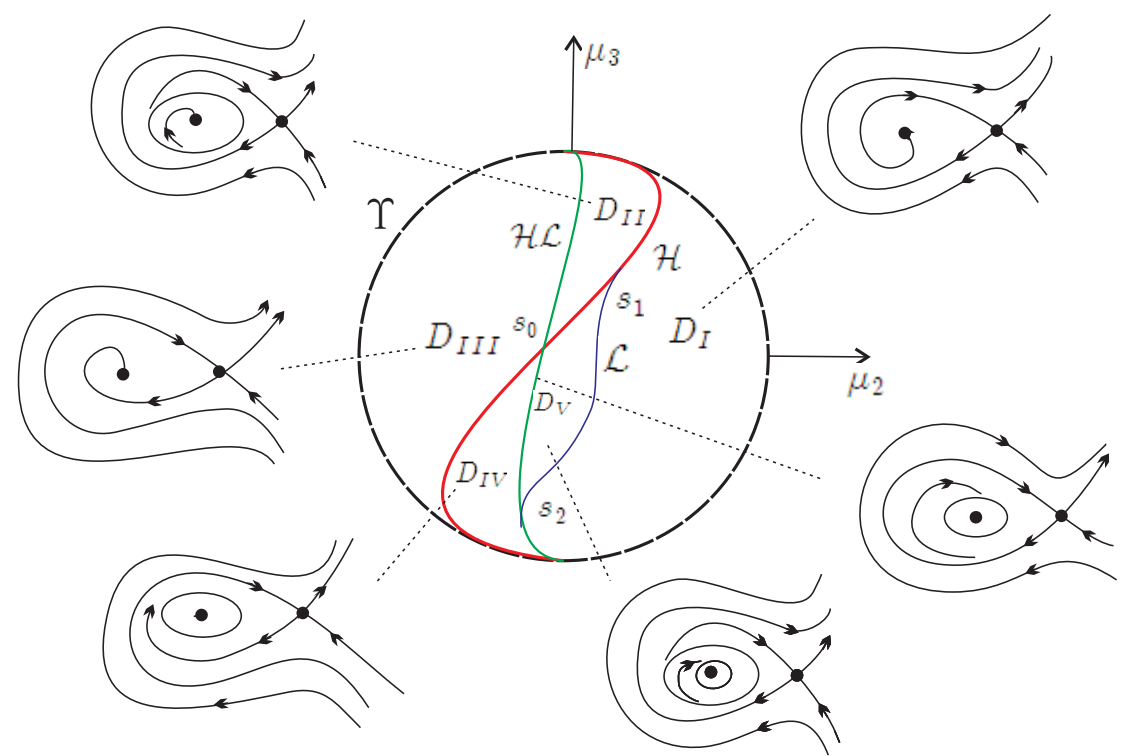

FIG. 3. Projections of $\mathcal{S} \mathcal{N}, \mathcal{H}, \mathcal{H} \mathcal{L}$, and $\mathcal{L}$ on the $\left(\mu_{2}, \mu_{3}\right)$-plane.

crosses $\mathcal{H}^{\prime}$, a homoclinic bifurcation near $E_{0}$ as $(A, p, m)$ crosses $\mathcal{H} \mathcal{L}^{\prime}$, and a coalescence of two limit cycles near $E_{0}$ as $(A, p, m)$ crosses $\mathcal{L}^{\prime}$.

The expressions of these bifurcation surfaces given in Theorem 5.2 can be computed by using (5.11) and (5.1). For example, consider $A_{0}=63.73, p_{0}=4.45, m_{0}=$ 1.01, and $q=1000$. The surface $\mathcal{H} \mathcal{L}^{\prime}$ is presented as

$$
\begin{aligned}
& \frac{5}{7}\left(0.003018260808 \epsilon_{2}+1.509170071 \epsilon_{3}\right) \Theta\left(\epsilon_{1}, \epsilon_{2}, \epsilon_{3}\right) \\
& +\frac{103}{77}\left(\Theta\left(\epsilon_{1}, \epsilon_{2}, \epsilon_{3}\right)\right)^{3}+o\left(\left|\left(\epsilon_{1}, \epsilon_{2}, \epsilon_{3}\right)\right|^{3 / 2}\right)=0,
\end{aligned}
$$

where $\Theta\left(\epsilon_{1}, \epsilon_{2}, \epsilon_{3}\right)=\sqrt{0.09569545272 \epsilon_{1}-0.003062992329 \epsilon_{2}-3.033146367 \epsilon_{3}}$. Parameter values are given by the expression where the homoclinic bifurcation occurs. Expressions of other bifurcation surfaces $\mathcal{S} \mathcal{N}^{\prime}, \mathcal{H}^{\prime}$, and $\mathcal{L}^{\prime}$ can be given similarly. For a better understanding of the bifurcation diagram, let us observe bifurcation surfaces $\mathcal{S N}, \mathcal{H}, \mathcal{H} \mathcal{L}$, and $\mathcal{L}$ in Figure 3. Since each of them is a cone with vertex at the origin (up to a homeomorphism in the parameter space), as in [7], it suffices to observe the bifurcation diagram in a small half ball $S_{\mu_{0}}=\left\{\left(\mu_{1}, \mu_{2}, \mu_{3}\right) \mid \mu_{1}^{2}+\mu_{2}^{2}+\mu_{3}^{2}<\mu_{0}, \mu_{1}<0\right\}$ for sufficiently small $\mu_{0}>0$ and project the diagram to the plane $\mathcal{S N}$ (i.e., $\mu_{1}=0$ ). As indicated in [7], projected on the disk $\Upsilon=S_{\mu_{0}} \cap \mathcal{S N}$, the curve $\mathcal{H}$ intersects the curve $\mathcal{H} \mathcal{L}$ at a point $s_{0}$ and $\mathcal{L}$ is tangent to curves $\mathcal{H}$ and $\mathcal{H} \mathcal{L}$ at two points $s_{1}$ and $s_{2}$, respectively. Thus the half ball $S_{\mu_{0}}$ is divided into five open regions $D_{j}(j=I, I I, \ldots, V)$, as shown in Figure 3. Let $D_{j}^{\prime}(j=I, I I, \ldots, V)$ be the corresponding regions in the $(A, p, m)$-space and $s_{0}^{\prime}, s_{1}^{\prime}, s_{2}^{\prime}$ be the corresponding intersection points, which can be calculated with (5.11). When $(A, p, m)$ lies in these regions, by Theorem 3.1, system (1.4) has two equilibria $E_{+}$and $E_{-}$in the interior of the first quadrant and $E_{-}$is 
TABLE 1

Qualitative properties for various parameters.

\begin{tabular}{|c|c|c|c|}
\hline$(A, p, m)$ & $\overline{E_{+}}$ & Limit cycles & Homoclinic orbits \\
\hline$D_{I}^{\prime}$ & unstable focus or node & no & no \\
\hline $\mathcal{H}^{\prime} \backslash\left\{s_{0}^{\prime} s_{1}^{\prime}\right\}$ & weak focus(order 1 ) & no & no \\
\hline$s_{1}^{\prime}$ & weak focus(order 2$)$ & no & no \\
\hline$s_{0}^{\prime} s_{1}^{\prime}$ & stable weak focus(order 1 ) & 1 (order 1$)$ & 0 \\
\hline$D_{U}^{\prime}$ & stable focus or node & 1 (order 1$)$ & no \\
\hline $\mathcal{H} \mathcal{L}^{\prime} \backslash\left\{s_{0}^{\prime} s_{2}^{\prime}\right\}$ & focus or node & no & $1($ order 1$)$ \\
\hline$s_{0}^{\prime}$ & stable weak focus (order 1 ) & no & 1 (order 1$)$ \\
\hline$\overline{D_{I U I}^{\prime}}$ & stable focus or node & no & no \\
\hline$\overline{D_{I V}^{\prime}}$ & unstable focus or node & 1 (order 1) & no \\
\hline$s_{2}^{\prime}$ & unstable focus or node & no & $1($ order 2$)$ \\
\hline$\widehat{s_{0}^{\prime} s_{2}^{\prime}}$ & unstable focus or node & $1($ order 1$)$ & $1($ order 1$)$ \\
\hline$D_{V}^{\prime}$ & unstable focus or node & $2($ order 1$)$ & no \\
\hline$\overline{\mathcal{L}^{\prime}}$ & unstable focus or node & 1 (order 2$)$ & 0 \\
\hline
\end{tabular}

always a saddle but $E_{+}$is either a focus or a node. Furthermore, by Lemma 4.1 and Theorem 5.2 together with the theorems in [7] and [25], we can list more detailed dynamical behaviors in Table 1, where $\widehat{s_{0}^{\prime} s_{1}^{\prime}}$ and $\widehat{s_{0}^{\prime} s_{2}^{\prime}}$ denote the parts of bifurcation surfaces determined by the arcs on $\mathcal{H}$ and $\mathcal{H L}$, respectively, as shown in Figure 3. More concretely, neither a limit cycle nor a homoclinic loop appears in $D_{I}^{\prime}$; a limit cycle arises as parameters go through $\mathcal{H}^{\prime}$ from $D_{I}^{\prime}$ to $D_{I I}^{\prime}$; the limit cycle expands, deforms into a homoclinic loop and finally breaks as parameters go through $\mathcal{H} \mathcal{L}^{\prime}$ from $D_{I I}^{\prime}$ to $D_{I I I}^{\prime}$; a limit cycle arises again as parameters go through $\mathcal{H}^{\prime}$ from $D_{I I I}^{\prime}$ to $D_{I V}^{\prime}$. By continuity, if parameters go through the part of $\mathcal{H} \mathcal{L}^{\prime}$ below $s_{2}^{\prime}$ and return to $D_{I}^{\prime}$ from $D_{I V}^{\prime}$, the limit cycle disappears; if parameters go from $D_{I V}^{\prime}$ and hit the $\operatorname{arc} \widehat{s_{0}^{\prime} s_{2}^{\prime}}$, the limit cycle coexists with a homoclinic loop. Furthermore, if parameters enter the region $D_{V}^{\prime}$, the limit cycle persists and another limit cycle arises as the homoclinic loop breaks, i.e., two cycles coexist.

6. Discussion. The existence of limit cycles in epidemic models can be used to explain oscillatory phenomena observed in the dynamics of some infectious diseases. One of the mechanisms by which epidemic models exhibit periodic oscillations is bifurcation, which occurs when the parameters vary. Early work on studying the dynamics of epidemic models focused on Hopf bifurcation, homoclinic bifurcation, or saddle-node bifurcation separately by using only one bifurcation parameter (Derrick and van den Driessche [6], Hethcote and van den Driessche [14], Liu et al. [17, 18]). Recent studies indicate that some epidemic models undergo codimension 2 bifurcations near degenerate equilibria; i.e., a Bogdanov-Takens bifurcation, which includes a Hopf bifurcation, a homocline bifurcation and a saddle-node bifurcation, can occur when two parameters vary near their critical values (Lizana and Rivero [19], Ruan and Wang [25], Alexander and Moghadas [1, 2], Moghadas [21], Wang [26]). It is interesting to notice that not only epidemic models with nonlinear incidence rates but also simple epidemic models with bilinear mass-action incidence rates can have complex dynamics such as the occurrence of Bogdanov-Takens bifurcations. For instance, Wang and Ruan [27] considered an epidemic model with a bilinear mass-action incidence rate and a constant removal rate of infectious individuals and showed that the model undergoes a sequence of bifurcations, including saddle-node bifurcation, subcritical Hopf bifurcation, and homoclinic bifurcation. 
In those epidemic models exhibiting Bogdanov-Takens bifurcations, periodic solutions can arise through a Hopf bifurcation for some parameter values and disappear through a homoclinic bifurcation for some other parameter values, but neither the existence of multiple limit cycles nor the coexistence of a limit cycle and a homoclinic loop is revealed. However, recent work (Alexander and Moghadas [1, 2], Liu, Hethcote, and Levin [17], Moghadas and Alexander [22], Ruan and Wang [25], Wang [26]) indicates that some epidemic models can have two limit cycles. One may expect that the appearance of two limit cycles is due to the fact that degenerate Hopf and degenerate Bogdanov-Takens bifurcations [4] may occur in such epidemic models as well. However, to the best of our knowledge, so far there is no such study on the degenerate Hopf bifurcation and degenerate Bogdanov-Takens bifurcation on epidemic models. One of the difficulties is the lack of general criteria in calculating the multiplicity of a weak focus (see Xiao and Zhu [30] for such a criterion for a predator-prey model; see also Ruan and Xiao [24]).

In this paper, we continued studying the dynamics of a simplified epidemic model (1.3) with a nonlinear incidence rate that was originally considered by Ruan and Wang [25] (see also Liu et al. [17, 18] and Hethcote and van den Driessche [14]). Under certain conditions Ruan and Wang [25] showed that the simplified model (1.3) undergoes a Bogdanov-Takens bifurcation; i.e., it exhibits saddle-node, Hopf, and homoclinic bifurcations. They also established the existence of none, one, or two limit cycles. In this paper, we first calculated the second order Liapunov value of the weak focus and proved that the maximal multiplicity of the weak focus is 2 by technically dealing with some complicated multivariable polynomials, which implies that at most two limit cycles can arise near the weak focus. Then, by reducing the determination of the sign for polynomials of higher degrees to revised sign lists, we re-established the uniqueness of the limit cycle. Finally, we reduced system (1.4) to a form of universal unfolding for a cusp of codimension 3 and showed the coexistence of limit cycles and homoclinic loops via a degenerate Bogdanov-Takens bifurcation.

The coexistence of limit cycles and homoclinic loops demonstrates that epidemic models with saturated incidence rates exhibit very different and complex dynamics. Furthermore, the results indicate that the dynamical behavior of the model is very sensitive to the initial densities of the susceptible and infectious individuals. When the initial values lie inside the homoclinic loop, the numbers of susceptible and infectious individuals fluctuate periodically about the endemic levels. Such periodic patterns will be helpful in designing control and intervention policies for the disease. When the initial values lie outside the homoclinic loop, the disease will die out even if there are two endemic equilibria (see Figure 3 ). This means that the disease can be controlled and eradicated even above the threshold.

To the best of our knowledge, this is the first time that a limit cycle and a homoclinic loop have been shown to coexist in a realistic epidemic model. Though we focused on a simple case of SIRS models with a specific saturated incidence rate, we believe that such rich and complex dynamics can occur in other epidemic models with general saturated incidence rates as well as other types of nonlinear incidence rates (Hethcote and van den Driessche [14], Liu et al. [17, 18]).

\section{Appendix.}

(A1) As claimed in section 4 , for each $j=1, \ldots, 13$, the polynomial $\varpi_{j}$ in variables 
$\alpha_{1}, \ldots, \alpha_{6}$, being the $j$ th principal minor of the matrix

$\operatorname{Discr}(\Psi)=\left[\begin{array}{lllllllllllll}\alpha_{0} & \alpha_{1} & \alpha_{2} & \alpha_{3} & \alpha_{4} & \alpha_{5} & \alpha_{6} & 0 & 0 & 0 & 0 & 0 & 0 \\ 0 & 6 \alpha_{0} & 5 \alpha_{1} & 4 \alpha_{2} & 3 \alpha_{3} & 2 \alpha_{4} & \alpha_{5} & 0 & 0 & 0 & 0 & 0 & 0 \\ 0 & \alpha_{0} & \alpha_{1} & \alpha_{2} & \alpha_{3} & \alpha_{4} & \alpha_{5} & \alpha_{6} & 0 & 0 & 0 & 0 & 0 \\ 0 & 0 & 6 \alpha_{0} & 5 \alpha_{1} & 4 \alpha_{2} & 3 \alpha_{3} & 2 \alpha_{4} & \alpha_{5} & 0 & 0 & 0 & 0 & 0 \\ 0 & 0 & \alpha_{0} & \alpha_{1} & \alpha_{2} & \alpha_{3} & \alpha_{4} & \alpha_{5} & \alpha_{6} & 0 & 0 & 0 & 0 \\ 0 & 0 & 0 & 6 \alpha_{0} & 5 \alpha_{1} & 4 \alpha_{2} & 3 \alpha_{3} & 2 \alpha_{4} & \alpha_{5} & 0 & 0 & 0 & 0 \\ 0 & 0 & 0 & \alpha_{0} & \alpha_{1} & \alpha_{2} & \alpha_{3} & \alpha_{4} & \alpha_{5} & \alpha_{6} & 0 & 0 & 0 \\ 0 & 0 & 0 & 0 & 6 \alpha_{0} & 5 \alpha_{1} & 4 \alpha_{2} & 3 \alpha_{3} & 2 \alpha_{4} & \alpha_{5} & 0 & 0 & 0 \\ 0 & 0 & 0 & 0 & \alpha_{0} & \alpha_{1} & \alpha_{2} & \alpha_{3} & \alpha_{4} & \alpha_{5} & \alpha_{6} & 0 & 0 \\ 0 & 0 & 0 & 0 & 0 & 6 \alpha_{0} & 5 \alpha_{1} & 4 \alpha_{2} & 3 \alpha_{3} & 2 \alpha_{4} & \alpha_{5} & 0 & 0 \\ 0 & 0 & 0 & 0 & 0 & \alpha_{0} & \alpha_{1} & \alpha_{2} & \alpha_{3} & \alpha_{4} & \alpha_{5} & \alpha_{6} & 0 \\ 0 & 0 & 0 & 0 & 0 & 0 & 6 \alpha_{0} & 5 \alpha_{1} & 4 \alpha_{2} & 3 \alpha_{3} & 2 \alpha_{4} & \alpha_{5} & 0 \\ 0 & 0 & 0 & 0 & 0 & 0 & \alpha_{0} & \alpha_{1} & \alpha_{2} & \alpha_{3} & \alpha_{4} & \alpha_{5} & \alpha_{6}\end{array}\right]$,

can be calculated directly with Maple software. For example, $\varpi_{1}=\alpha_{0}, \varpi_{2}=$ $6 \alpha_{0}^{2}, \varpi_{3}=\alpha_{0}^{2} \alpha_{1}, \varpi_{4}=-\alpha_{0}^{2}\left(-5 \alpha_{1}^{2}+12 \alpha_{2} \alpha_{0}\right), \varpi_{5}=\alpha_{0}^{2}\left(\alpha_{1}^{2} \alpha_{2}-4 \alpha_{0} \alpha_{2}^{2}+3 \alpha_{0} \alpha_{3} \alpha_{1}\right)$, $\varpi_{6}=2 \alpha_{0}^{2}\left(24 \alpha_{0}^{2} \alpha_{4} \alpha_{2}-27 \alpha_{0}^{2} \alpha_{3}^{2}-8 \alpha_{0} \alpha_{2}^{3}+24 \alpha_{0} \alpha_{3} \alpha_{1} \alpha_{2}-10 \alpha_{0} \alpha_{4} \alpha_{1}^{2}+2 \alpha_{2}^{2} \alpha_{1}^{2}-5 \alpha_{3} \alpha_{1}^{3}\right)$. The expressions for others will be much longer.

(A2) The polynomials $\vartheta_{j}, \iota_{i j}, \omega_{j}$ claimed in (5.4), $a_{i j}$ and $b_{i j}$ in (5.6), $c_{i j}$ and $F_{3}$ in (5.7), and $d_{i j}$ in (5.9) can be calculated directly with Maple software. Except for

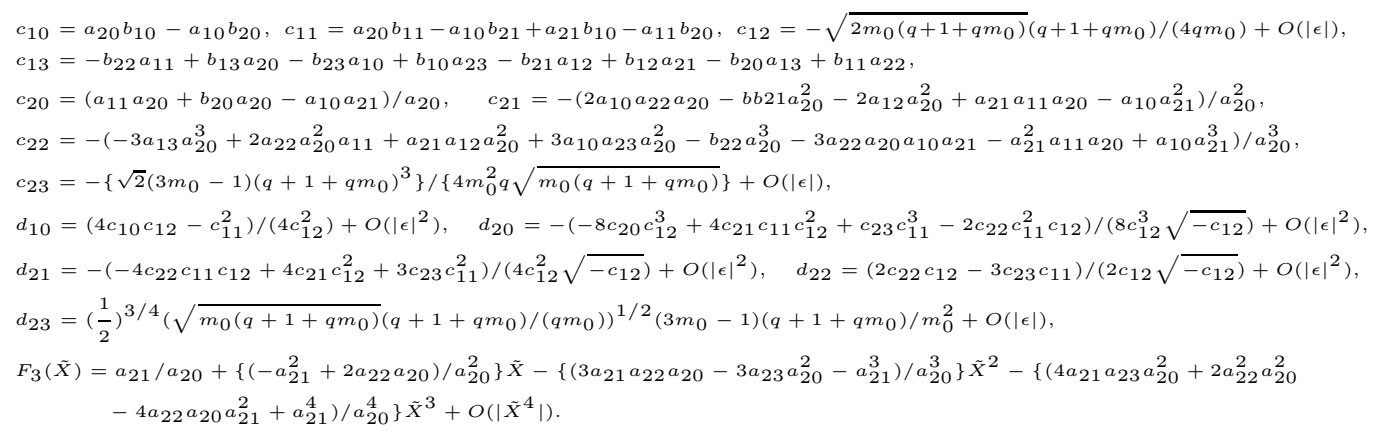

The other polynomials have long expressions. Their presentations and the Maple scripts are available upon request.

Acknowledgment. The authors are very grateful to the referees for their helpful comments and suggestions.

\section{REFERENCES}

[1] M. E. Alexander and S. M. Moghadas, Periodicity in an epidemic model with a generalized non-linear incidence, Math. Biosci., 189 (2004), pp. 75-96.

[2] M. E. Alexander And S. M. Moghadas, Bifurcation analysis of an SIRS epidemic model with generalized incidence, SIAM J. Appl. Math., 65 (2005), pp. 1794-1816.

[3] R. M. Anderson And R. M. May, Infectious Diseases of Humans: Dynamics and Control, Oxford University Press, Oxford, UK, 1992.

[4] S. M. Baer, B. W. Kooi, Y. A. Kuznetsov, and H. R. Thieme, Multiparametric bifurcation analysis of a basic two-stage population model, SIAM J. Appl. Math., 66 (2006), pp. 13391365.

[5] V. Capasso And G. Serio, A generalization of the Kermack-Mckendrick deterministic epidemic model, Math. Biosci., 42 (1978), pp. 43-61.

[6] W. R. DERrick AND P. VAN DEN Driessche, Homoclinic orbits in a disease transmission model with nonlinear incidence and nonconstant population, Discrete Contin. Dyn. Syst. Ser. B, 3 (2003), pp. 299-309. 
[7] F. Dumortier, R. Roussarie, and J. Sotomayor, Generic 3-parameter families of vector fields on the plane, unfolding a singularity with nilpotent linear part. The cusp case of codimension 3, Ergodic Theory Dynam. Systems, 7 (1987), pp. 375-413.

[8] M. G. M. Gomes, A. Margheri, G. F. Medley, and C. Rebelo, Dynamical behaviour of epidemiological models with sub-optimal immunity and nonlinear incidence, J. Math. Biol., 51 (2005), pp. 414-430.

[9] J. K. Hale, Ordinary Differential Equations, 2nd ed., Wiley-Interscience, New York, 1980.

[10] H. W. Нетнсоте, A thousand and one epidemic models, in Frontiers in Theoretical Biology, S. A. Levin, ed., Lecture Notes in Biomath. 100, Springer-Verlag, Berlin, 1994, pp. 504-515.

[11] H. W. Heтнсоте, The mathematics of infectious diseases, SIAM Rev., 42 (2000), pp. 599-653.

[12] H. W. Hethcote and S. A. Levin, Periodicity in epidemiological models, in Applied Mathematical Biology, Biomath. Texts 18, S. A. Levin, T. G. Hallam and L. J. Gross, eds., Springer-Verlag, New York, 1989, pp. 193-211.

[13] H. W. Hethcote, H. W. Stech, and P. van den Driessche, Periodicity and stability in epidemic models: A survey, in Differential Equations and Applications in Ecology, Epidemics and Population Problems, S. N. Busenberg and K. L. Cook, eds., Academic Press, New York, 1981, pp. 65-82.

[14] H. W. HethCote And P. VAn Den Driessche, Some epidemiological models with nonlinear incidence, J. Math. Biol., 29 (1991), pp. 271-287.

[15] R. E. Kooij And A. Zegeling, A predator-prey model with Ivlev's functional response, J. Math. Anal. Appl., 198 (1996), pp. 473-489.

[16] C.-Z. Li And C. Rousseau, A system with three cycles appearing in a Hopf bifurcation and dying in a homoclinic bifurcation: The cusp of order 4, J. Differential Equations, 23 (1986), pp. 187-204.

[17] W. Liu, H. W. Hethсote, and S. A. Levin, Dynamical behavior of epidemiological models with non-linear incidence rate, J. Math. Biol., 25 (1987), pp. 359-380.

[18] W. Liu, S. A. Levin, AND Y. IwaSA, Influence of nonlinear incidence rate upon the behavior of SIRS epidemiological models, J. Math. Biol., 23 (1986), pp. 187-204.

[19] M. Lizana And J. Rivero, Multiparametric bifurcations for a model in epidemiology, J. Math. Biol., 35 (1996), pp. 21-36.

[20] N. G. LlOYD, Limit cycles of polynomial systems - some recent developments, in New Directions in Dynamical Systems, T. Bedford and J. Swift, eds., LMS Lect. Notes 127, Cambridge University Press, Cambridge, UK, 1988, pp. 192-238.

[21] S. M. Moghadas, Analysis of an epidemic model with bistable equilibria using the Poincaré index, Appl. Math. Comput., 149 (2004), pp. 689-702.

[22] S. M. Moghadas And M. E. Alexander, Bifurcations of an epidemic model with non-linear incidence and infection-dependent removal rate, Math. Med. Biol., 23 (2006), pp. 231-254.

[23] R. R. Regoes, D. Ebert, and S. Bonhoeffer, Dose-dependent infection rates of parasites produce the Allee effect in epidemiology, Proc. Roy. Soc. London Ser. B, 269 (2002), pp. $271-279$.

[24] S. RUAN AND D. XIAO, Global analysis in a predator-prey system with nonmonotonic functional response, SIAM J. Appl. Math., 61 (2001), pp. 1445-1472.

[25] S. Ruan And W. Wang, Dynamical behaviour of an epidemic model with a nonlinear incidence rate, J. Differential Equations, 188 (2003), pp. 135-163.

[26] W. WAng, Epidemic models with nonlinear infection forces, Math. Biosci. Engrg., 3 (2006), pp. 267-279.

[27] W. WANG AND S. RuAn, Bifurcations in an epidemic model with constant removal rate of the infectives, J. Math. Anal. Appl., 291 (2004), pp. 775-793.

[28] D. XIAO AND S. RuAN, Global analysis of an epidemic model with nonmonotone incidence rate, Math. Biosci., 208 (2007), pp. 419-429.

[29] D. XIAO AND Z.-F. Zhang, On the uniqueness and nonexistence of limit cycles for predatorprey systems, Nonlinearity, 16 (2003), pp. 1185-1201.

[30] D. XIAO AND H. ZHU, Multiple focus and Hopf bifurcations in a predator-prey system with nonmonotonic functional response, SIAM J. Appl. Math., 66 (2006), pp. 802-819.

[31] L. YANG, Recent advances on determining the number of real roots of parametric polynomials, J. Symbolic Comput. 28 (1999), pp. 225-242.

[32] Z.-F. Zhang, T.-R. Ding, W.-Z. Huang, and Z.-X. Dong, Qualitative Theory of Differential Equations, AMS, Providence, RI, 1992.

Copyright $@$ by SIAM. Unauthorized reproduction of this article is prohibited. 